九州大学学術情報リポジトリ

Kyushu University Institutional Repository

\title{
An extensive direct assay in biological assay
}

Asano, Choichiro

Shionogi Pharamaceutical Co. | Kyushu University

https://doi.org/10.5109/12997

出版情報 : 統計数理研究. 10 (1/2)，pp. 1-16，1961-10. Research Association of Statistical Sciences

バージョン :

権利関係 : 


\title{
AN EXTENSIVE DIRECT ASSAY IN BIOLOGICAL ASSAY
}

\author{
By \\ Chooichiro Asano
}

\section{§. Introduction}

The principle of a direct assay is that doses of a standard and a test preparation to produce a specified response are "directly" measured and the assay depends upon the possibility of measuring the dose just needed and is independent with measuring the sufficiently large dose to produce a specified response. Thus the potency of the test preparation relative to the standard is estimated as a ratio between these critical doses on the basis of the definition of relative potency, that is, this potency is an amount of the standard equivalent in effect to one unit of the test. Indeed, from these situations of assay, the current trials of the direct assay for both preparations have been made on a certain number of subjects respectively and then the estimate of the relative potency has been made it a rule to calculate a ratio between both averages of the respective observations.

In this paper, a new extensive method of the direct assay is proposed by appling certain functional relationship in effects of the standard preparation and of the test preparation denoted by their critical doses and there the doses administered are planned out adopting some various amounts of the standard preparation and also of the test preparation, as described for details in section 2 , in order to get a better precise expectation and a test of the relative potency and/or to perform an assay on fewer numbers of subjects under a demand with certain precision of an inference of relative potency than those of the current trials.

Section 2 of this paper is concerned with the description of our present method and formulation of the assay, section 3 is concerned with the estimation of the relative potency of test preparation and section 4 is with the test for the relative potency. In section 5 , we give the numerical illustrations in the assay of short acting narcotics.

\section{§ 2. Method and formulation of our assay}

The typical method of our present direct assay for the potency of a test preparation relative to a standard is that a certain dose of standard preparation is first imposed as a preliminary operation and secondly a dose 
of test preparation is infused into a subject until a specified response is observed. This is repeated on several subjects at any rate of doses of two preparations or, at least, at two rates for two preparations.

The critical dose will generally vary at each time of trials and it is important that the variation of response depends upon the homogeneity of the subjects, careful control of environment, the history of bred stock of subjects as in other many biological assays. In some cases, regardless these considerations, however, we may find a fluctuation and now shall regard that of the critical doses as that caused by administration of the dose of each preparation, for instance, in case assayed by using large animals taken care of the homogeneity of the response. From other view-point, this situation may also be applied in case when the fluctuation caused by administration of the dose is larger considerably than that depending upon the homogeneity of the subjects.

Then the model of the present assay is expressed in the following ways.

Let a parameter $C$ be a true critical dose needed to produce just a specified response and let $\xi_{i}$ and $\eta_{i}$ be two parameters denoted the true doses administered of test preparation and standard preparation respectively in any $i$-th trial, $(i=1,2, \ldots, n)$. Then the following functional relationship concerning to a critical dose is obtained by

$$
C=\eta_{i}+\beta^{*} \xi_{i}, \quad(i=1,2, \ldots, n),
$$

where $\beta^{*}$ is defined as the relative potency, that is, the potency of the test preparation relative to a standard. In the case we now consider, the parameter $\eta_{i}$ and $\xi_{i}$ are subject to error:

$$
\begin{aligned}
& y_{i}=\eta_{i}+v_{i}, \\
& x_{i}=\xi_{i}+u_{i},
\end{aligned}
$$

where $v_{i}$ and $u_{i}$ are random variables drawn from two independent normal distributions $N\left(0, \sigma_{v}{ }^{2}\right)$ and $N\left(0, \sigma_{u}{ }^{2}\right)$ respectively. And here the size of the fluctiations $\sigma_{v}{ }^{2}$ and $\sigma_{u}{ }^{2}$ caused by the administration of each preparation may or not may be considered to be equal, namely $\lambda=1$, each other in the present assay. In some dilution assay, however, both sizes of the fluctuation may be unequal. Therefore we shall put that $\sigma_{u}{ }^{2} / \sigma_{v}{ }^{2}=\lambda$ as a general case and shall assume that the value of $\lambda$ is known to us by a previous information.

Furthermore we shall now assume essentially the important proposition for the present assay design that the dose-levels are chosen to be expanded sufficiently as large as available for the linearity of the dose-response curve, or that the size $n$ of the assay planned previously is to be such as the sum of square $\sum_{i=1}^{n}\left(\xi_{i}-\bar{\xi}_{.}\right)^{2}$ is considerably large in comparison with the suggestive value of $\sigma^{2}$. And these circumstances are the same as the proposi- 
tion for the improvement of the precision of an estimated line in the case of ordinary regression analysis and may be natural.

Thus the present problem of our extensive direct assay is reduced to estimate and to test the relative potency $\beta^{*}$ on a test preparation.

\section{§3. Estimation of the relative potency}

Let us consider the situation where we are provided with the pair of observations, $O_{n}:\left(x_{i}, y_{i}\right),(i=1,2, \ldots, n)$, and assume that $x_{i}$ and $y_{i}$ are distributed in $N\left(\xi_{i}, \lambda \sigma^{2}\right)$ and in $N\left(\eta_{i}, \sigma^{2}\right)$ respectively, where the value of $\lambda$ is known to us by a previous information but has not necessarily +1 , however, the value of $\lambda$ is unknown to us. And let us assume afresh that there is a following functional relationship among $\eta_{i}$ and $\xi_{i}$,

$$
\eta_{i}=\mu+\beta\left(\xi_{i}-\bar{\xi}_{.}\right) \quad, \quad(i=1,2, \ldots, n),
$$

where $\beta$ and $\mu-\beta \bar{\xi}$. are corresponding with $-\beta^{*}$ and $C$ respectively in (2.1), and where $\beta \neq 0$ for reasons of that our aim performed the direct assay has no sense in case when the relative efficiency of test preparation is considered as zero exactly, and assume that errors in $x_{i}$ and $y_{i}$ are mutually independent.

Hence a parameter $\Delta_{x y}$ defined as a correlation coefficient among $x_{i}$ and $\boldsymbol{y}_{i},(i=1,2, \ldots, n)$, is given as follows:

$$
\Delta_{x y}=S_{\xi \eta} / \sqrt{\left(S_{\xi \xi}+\lambda \sigma^{2}\right)\left(\overline{\left.S_{\eta n}+\sigma^{2}\right)}\right.},
$$

where we put

and

$$
\begin{aligned}
& S_{\xi \xi}=\sum_{i=1}^{n}\left(\xi_{i}-\bar{\xi}_{.}\right)^{2} / n, S_{\eta \eta}=\sum\left(\eta_{i}-\bar{\eta}_{.}\right)^{2} / n=\beta^{2} S_{\xi \xi}, \\
& S_{\xi \eta}=\sum\left(\xi_{i}-\bar{\xi}_{.}\right)\left(\eta_{i}-\bar{\eta}_{.}\right) / n=\beta S_{\xi \xi} .
\end{aligned}
$$

\subsection{Point estimation}

In order to obtain a point estimation of relative potency $\beta$, we obtain a likelihood function

$$
\left(2 \pi \sqrt{\lambda} \sigma^{2}\right)^{-n} \exp \left[-\frac{1}{2 \sigma^{2}} \sum_{i}\left\{\left(x_{i}-\xi_{i}\right)^{2} / \lambda+\left(y_{i}-\mu-\beta\left(\xi_{i}-\bar{\xi}_{\cdot}\right)\right)^{2}\right\}\right],
$$

and the maximum likelihood estimate of $\beta$ is given as follows:

$$
\hat{\beta}=\hat{\theta} \pm \sqrt{\hat{\theta}^{2}+\lambda^{-1}},
$$

where

$$
\begin{aligned}
& \hat{\theta}=\left\{\sum_{i}\left(y_{i}-\bar{y}\right)^{2}-\lambda^{-1} \sum_{i}\left(x_{i}-\bar{x}\right)^{2}\right\} / 2 \sum\left(x_{i}-\bar{x}\right)\left(y_{i}-\bar{y}\right), \\
& \hat{\mu}=\bar{y}, \quad \hat{\xi}=\bar{x}, \quad \hat{\xi}_{i}=\left\{x_{i}+\hat{\beta} \lambda\left(y_{i}-\bar{y}+\hat{\beta} \bar{x}\right)\right\} /\left(1+\hat{\beta}^{2} \lambda\right),
\end{aligned}
$$




$$
\hat{\sigma}^{2}=\sum_{i}\left\{\left(x_{i}-\hat{\xi}_{i}\right)^{2} / \lambda+\left(y_{i}-\hat{\eta}_{i}\right)^{2}\right\} / 2 n,
$$

and the sign \pm of $(3.5)$ is taken corresponding as $\sum_{i}\left(x_{i}-\bar{x}\right)\left(y_{i}-\bar{y}\right)$ is positive or negative.

Specially when $\lambda$ is regarded as zero, the estimate of $\beta$ is given at a regression situation by

$$
\hat{\beta}=\sum\left(x_{i}-\bar{x}\right)\left(y_{i}-\bar{y}\right) / \sum\left(x_{i}-\bar{x}\right)^{2}, \hat{\sigma}^{2}=\sum\left\{\left(y_{i}-\bar{y}\right)-\hat{\beta}\left(x_{i}-\bar{x}\right)\right\}^{2} /(n-2),
$$

and specially when $\lambda$ is infinite we have $\hat{\beta}$ which is substituted $(y, x)$ for $(x, y)$ in $(3.7)$ as a regression of $x$ on $y$. For the general case of the value of $\lambda$ between zero and infinity, the line (3.1) lies between these two extremes and the statistic $\hat{\beta}$ is biassed but is consistent. In particular if $\lambda=1$, the line, that is, a diagonal regression takes the direction of the first principal component.

\subsection{Expectation, variance, skewness and kurtosis of $\hat{\beta}$}

In order to make arrangements for some properties of the statistic $\hat{\beta}$, we may and now shall first seek several moments of $\hat{\theta}$ instead of $\hat{\beta}$ and secondly we shall evaluate the expectation, variance, skewness and kurtosis of $\hat{\beta}$ as a certain approximation, because of that the statistic $\hat{\theta}$ has one-toone correspondence with statistic $\hat{\beta}$.

The moments about an origin of $\hat{\theta}$ may be calculated in the following way:

$$
\begin{array}{r}
\mu_{i}=E\left\{\hat{\theta}^{i}\right\}=\int_{0}^{\infty} \int_{0}^{\infty}\left[\int_{-\sqrt{m_{20} m_{02}}}^{\sqrt{m_{20} m_{02}}} \hat{\theta}^{i}\left(m_{20}, m_{02}, m_{11}\right) f_{n-1}\left(m_{20}, m_{02}, m_{11}\right) d m_{11}\right] \\
d m_{20} d m_{02},
\end{array}
$$

where $f\left(m_{20}, m_{02}, m_{11}\right)$ is the density function of two component Wishart distribution which is now shown as

$$
\begin{aligned}
f_{n-1}\left(m_{20}, m_{02}, m_{11}\right)= & \left(\sqrt{\lambda\left(1-\rho^{2}\right)} \sigma^{2} / n\right)^{-(n-1)}\left(m_{20} m_{02}-m_{11}\right)^{\frac{n-4}{2}} \\
& \cdot \exp \left[-\frac{n}{2\left(1-\rho^{2}\right) \sigma^{2}}\left\{\frac{m_{20}}{\lambda}-2 \rho \frac{m_{11}}{\sqrt{\lambda}}+m_{02}\right\}\right] / 4 \pi(n-3) !
\end{aligned}
$$

and

$$
m_{20}=\sum\left(u_{i}-\bar{u}_{.}\right)^{2} / n, m_{02}=\sum\left(v_{i}-\bar{v}_{.}\right)^{2} / n, m_{11}=\sum\left(u_{i}-\bar{u}_{.}\right)\left(v_{i}-\bar{v}_{.}\right) / n
$$

Performing the integral calculations of (3.8), the values are shown formally for $i=1,2,3$ and 4 as follows: 


$$
\begin{aligned}
E\{\hat{\theta}\}= & \frac{\left(\beta^{2}-\lambda^{-1}\right)}{2 \beta}\left(1-\rho^{2}\right) \sum_{\substack{i, \nu=0 \\
(i+\nu \text { ieven })}}^{\frac{n-1}{2}}(-1)^{i} \frac{(2 \rho)^{\nu}}{\nu !} \frac{\Gamma\left(\frac{n+i+\nu-1}{2}\right) \Gamma\left(\frac{i+\nu+1}{2}\right)}{\sqrt{\pi} \Gamma\left(\frac{n-1}{2}\right)} \\
& \cdot\left(\frac{2 \sqrt{\lambda}\left(1-\rho^{2}\right) \sigma^{2}}{n \beta S_{\xi \xi}}\right)^{i},
\end{aligned}
$$

(3.11) $E\left\{\hat{\theta}^{2}\right\}=\left(1-\rho^{2}\right) \sum_{\substack{i, \nu \\(i+\nu \text { :even })}}^{\frac{n-1}{2}}(-1) \frac{\left(\begin{array}{c}i+1 \\ i\end{array}\right)(2 \rho)^{\nu}}{\nu !} \frac{\Gamma\left(\frac{n+i+\nu-1}{2}\right) \Gamma\left(\frac{i+\nu+1}{2}\right)}{\sqrt{\pi} \Gamma\left(\frac{n-1}{2}\right)}$

$$
\begin{aligned}
& \cdot\left(\frac{2 \sqrt{\lambda}\left(1-\rho^{2}\right) \sigma^{2}}{n \beta S_{\xi \xi}}\right)^{i}\left\{\left(\frac{\beta^{2}-\lambda^{-1}}{2 \beta}\right)^{2}+2 \frac{\Gamma\left(\frac{n+i+\nu+1}{2}\right)}{\Gamma\left(\frac{n+i+\nu-1}{2}\right)}\left(\frac{\left(1-\rho^{2}\right) \sigma^{2}}{n \beta S_{\xi \xi}}\right)^{2}\right\}, \\
& E\left\{\hat{\theta}^{3}\right\}=\frac{\left(\beta^{2}-\lambda^{-1}\right)}{2 \beta}\left(1-\rho^{2}\right)^{\frac{n-1}{2}} \sum_{\substack{i, \nu \\
(i+\nu \text { :even })}}(-1) \frac{\left(\begin{array}{c}
i+2 \\
i
\end{array}\right)(2 \rho)^{\nu}}{\nu !} \frac{\left(\frac{n+i+\nu-1}{2}\right) \Gamma\left(\frac{i+\nu+1}{2}\right)}{\sqrt{\pi} \Gamma\left(\frac{n-1}{2}\right)} \\
& \cdot\left(\frac{2 \sqrt{\bar{\lambda}}\left(1-\rho^{2}\right) \sigma^{2}}{n \beta S_{\xi \xi}}\right)^{i}\left\{\left(\frac{\beta^{2}-\lambda^{-1}}{2 \beta}\right)^{2}+6 \frac{\Gamma\left(\frac{n+i+\nu+1}{2}\right)}{\Gamma\left(\frac{n+i+\nu-1}{2}\right)}\left(\frac{\left(1-\rho^{2}\right) \sigma^{2}}{n \beta S_{\xi \xi}}\right)^{2}\right\} \text {, }
\end{aligned}
$$

and

$$
\begin{gathered}
E\left\{\hat{\theta}^{4}\right\}=\left(1-\rho^{2}\right)^{\frac{n-1}{2}} \Sigma(-1) \frac{\left(\begin{array}{c}
i+3 \\
i
\end{array}\right)(2 \rho)^{\nu}}{\nu !} \frac{\Gamma\left(\frac{n+i+\nu-1}{2} \Gamma\left(\frac{i+\nu+1}{2}\right)\right.}{\sqrt{\pi} \Gamma\left(\frac{n-1}{2}\right)} \\
\cdot\left(\frac{2 \sqrt{\lambda}\left(1-\rho^{2}\right) \sigma^{2}}{n \beta S_{\xi \xi}}\right)^{i}\left\{\left(\frac{\beta^{2}-\lambda^{-1}}{2 \beta}\right)^{4}+12 \frac{\Gamma\left(\frac{n+i+\nu+1}{2}\right)}{\Gamma\left(\frac{n+i+\nu-1}{2}\right)}\left(\frac{\beta^{2}-\lambda^{-1}}{2 \beta}\right)^{2}\left(\frac{\left(1-\rho^{2}\right) \sigma^{2}}{n \beta S_{\xi \xi}}\right)^{2}\right. \\
\left.+12 \frac{\Gamma\left(\frac{n+i+\nu+1}{2}\right)}{\Gamma\left(\frac{n+i+\nu-1}{2}\right)}\left(\frac{\left(1-\rho^{2}\right) \sigma^{2}}{n \beta S_{\xi \xi}}\right)^{4}\right\},
\end{gathered}
$$

where we assume naturally that $\beta \Rightarrow 0$. In the current case of direct assay, we may and now shall adopt approximately that $v^{-}\left(1-\rho^{2}\right) \sigma^{2} / n \beta S_{\xi \xi} \ll 1$ and $\left(1-\rho^{2}\right)^{(n-1) / 2}=1$. Indeed we assume that $\rho=0$ at (3.2). Then the values of (3.11) and the values of moments about the mean may be expressed 
sufficiently with the precise approximation in the following way.

(a) Moments of $\hat{\theta}$ about an origin.

$$
\begin{aligned}
& \mu_{1}^{\prime}=\frac{\beta^{2}-\lambda^{-1}}{2 \beta}, \\
& \mu_{2}^{\prime}=\left(\frac{\beta^{2}-\lambda^{-1}}{2 \beta}\right)^{2}+(n-1)\left(\frac{\sigma^{2}}{n \beta S_{\xi \xi}}\right)^{2}, \\
& \mu_{3}^{\prime}=\left(\frac{\beta^{2}-\lambda^{-1}}{2 \beta}\right)^{3}+3(n-1)\left(\frac{\beta^{2}-\lambda^{-1}}{2 \beta}\right)\left(\frac{\sigma^{2}}{n \beta S_{\xi \xi}}\right)^{2}, \\
& \mu_{4}^{\prime}=\left(\frac{\beta^{2}-\lambda^{-1}}{2 \beta}\right)^{4}+6(n-1)\left(\frac{\beta^{2}-\lambda^{-1}}{2 \beta}\right)^{2}\left(\frac{\sigma^{2}}{n \beta S_{\xi \xi}}\right)^{2}+3\left(n^{2}-1\right)\left(\frac{\sigma^{2}}{n \beta S_{\xi \xi}^{-}}\right)^{4},
\end{aligned}
$$

and

$$
\mu_{-1}^{\prime}=\frac{2 \beta}{\beta^{2}-\lambda^{-1}} .
$$

(b) Moments of $\hat{\theta}$ about the mean.

and

$$
\begin{aligned}
& \mu_{2}=(n-1)\left(\frac{\sigma^{2}}{n \beta S_{\xi \xi}}\right)^{2}, \\
& \mu_{3}=0,
\end{aligned}
$$

$$
\mu_{4}=3\left(n^{2}-1\right)\left(\frac{\sigma^{2}}{n \beta S_{\xi \xi}}\right)^{4} .
$$

Furthermore using these moments, skewness $\gamma_{1}$ and kurtosis $\gamma_{2}$ are calculated as follows:

$$
\begin{aligned}
& r_{1}=0, \\
& r_{2}\left(=\frac{\mu_{4}}{\mu_{2}^{2}}-3\right)=\frac{6}{n-1} .
\end{aligned}
$$

On the basis of the above results concerning to $\hat{\theta}$, the expectation and the variance of $\hat{\beta}$ for any $\lambda$ between zero and infinity may be given approximately in the following way.

(A) If we may assume $\theta^{2}>\lambda^{-1}$, then a parameter $\beta$ and a statistic $\hat{\beta}$ defined by (3.5) are regarded as

$$
\begin{aligned}
& \beta=\theta+\sqrt{\theta^{2}+\lambda^{-1}}=\theta+\theta\left\{1+\frac{\left(\frac{\lambda^{-1}}{\theta^{2}}\right)}{2}-\frac{\left(\frac{\lambda^{-1}}{\theta^{2}}\right)^{2}}{8}+\frac{\left(\frac{\lambda^{-1}}{\theta^{2}}\right)^{3}}{16}+\cdots\right\}=2 \theta+\frac{\lambda^{-1}}{2 \theta}, \\
& \hat{\beta}=2(\theta+e)+\frac{\lambda^{-1}}{2(\theta+e)} \div 2 \theta+\frac{\lambda^{-1}}{2 \theta}+e\left(2-\frac{\lambda^{-1}}{2 \theta^{2}}\right), \quad e=\frac{\sigma_{e}}{\sqrt{n}} Z,
\end{aligned}
$$

respectively, where $Z$ indicates a random variable distributing in a normal 
population $N(0,1)$ and where $\theta=\left(\beta^{2}-\lambda^{-1}\right) / 2 \beta$ and $\sigma_{e}^{2}=\frac{n-1}{n}\left(\frac{\sigma^{2}}{\beta S_{\xi \xi}}\right)^{2}$. Thus the expectation and the variance of $\hat{\beta}$ are obtained approximately as follows :

(3.16) $E(\hat{\beta}) \doteqdot 2 \theta+\frac{\lambda^{-1}}{2 \theta}$,

$$
V(\hat{\beta}) \fallingdotseq E\left\{(\hat{\beta}-\beta)^{2}\right\}=E\left\{\left(2-\frac{\lambda^{-1}}{2 \theta^{2}}\right)^{2} e^{2}\right\}=\left(2-\frac{\lambda^{-1}}{2 \theta^{2}}\right)^{2} \sigma^{2} / n
$$

(B) Otherwise if we may assume $\theta^{2}<\lambda^{-1}$, then $\beta$ and $\hat{\beta}$ defined by (3.5) are regarded as

$$
\begin{aligned}
& \beta \doteqdot \lambda^{-1 / 2}\left\{1+\frac{\theta}{\lambda^{-1 / 2}}+\frac{\theta^{2}}{2 \lambda^{-1}}-\frac{\theta^{4}}{8 \lambda^{-2}}+\cdots\right\} \div \lambda^{-1 / 2}+\theta, \\
& \hat{\beta} \fallingdotseq \lambda^{-1 / 2}+\theta+e,
\end{aligned}
$$

respectively, and the expectation and the variance of $\hat{\beta}$ are obtained approximately as follows:

$$
\begin{aligned}
& E\{\hat{\beta}\} \doteqdot \lambda^{-1 / 2}+\theta, \\
& V\{\hat{\beta}\} \fallingdotseq \sigma_{e}^{2} / n .
\end{aligned}
$$

\subsection{Interval estimation of the relative potency}

Now, instead of making the confidence limits $\hat{\beta}_{U}$ and $\hat{\beta}_{U}$ of $\beta$, we shall make the limits $\hat{\theta}_{U}$ and $\hat{\theta}_{L}$ of $\theta$ on the basis of the one-to-one correspondence between the $\hat{\beta}$ and the $\hat{\theta}$. Thus we may obtain mediately the $\hat{\beta}_{V}$ and $\hat{\beta}_{L}$ by the expression of $\hat{\theta}_{C}$ and $\hat{\theta}_{L}$.

Since the properties, however, shown by (3.11) of the estimate $\hat{\theta}$ are unessencially the excessive complication regardless as the circumstances of that $S_{\xi \xi} \gg m_{11}$ and hence $\left(S_{\xi \xi}+m_{11}\right)^{-1} \fallingdotseq S_{\xi \xi}^{-1}$ for the assumption $\rho=0$, namely, of that the conclusion negrecting the $m_{11}$ may be established, we may and now shall avoid the complication caused by the original $\hat{\theta}$ and, inspecting the moments (3.11), (3.12) and (3.13) of the estimate of $\theta$, we shall consider afresh the estimate $\hat{\theta}$ like defined by

$$
\hat{\theta}=\frac{\beta^{2}-\lambda^{-1}}{2 \beta}+\frac{m_{02}-\lambda^{-1} m_{20}}{2 \beta S_{\xi \xi}},
$$

with satisfaction for the practical use. Then $S^{\prime}$ defined by $n \beta S_{\xi \xi \hat{\theta}}$ is distributed in the non-central $\chi^{2}$-distribution with the degrees of freedom $\nu^{\prime}$ and the non-centricity parameter $\delta$, where we put that

$$
\nu^{\prime}=2(n-1) \text { and } \delta=n\left(\beta^{2}-\lambda^{-1}\right) S_{\xi \xi} / 2 \sigma^{2} .
$$

Furthermore, for the sake of Patnaik's approximation [1], this distribu- 
tion may be changed for an originary $\chi^{2}$-distribution with the degrees of freedom $\nu$ by substituting $S$ defined by $S^{\prime} / C$ for $S^{\prime}$, where

$$
\nu=\nu^{\prime}+4 \delta^{2} /\left(\nu^{\prime}+4 \delta\right) \text { and } C=1+\delta /(n-1+\delta) .
$$

Hence the confidence limits concerning to a parameter of $S$ may be expressed by

$$
\operatorname{Pr}\left\{S / \chi_{\nu}^{2}\left(\frac{\alpha}{2}\right)<E(S)<S / \chi_{\nu}^{2}\left(1-\frac{\alpha}{2}\right)\right\}=1-\alpha,
$$

where $\chi_{\nu}^{2}(\alpha / 2)$ and $\chi_{\nu}^{2}(1-\alpha / 2)$ are defined by the values such that

$$
\operatorname{Pr}\left\{\chi_{\nu}^{2}\left(\frac{\alpha}{2}\right)<\chi_{\nu}^{2}\right\}=\alpha / 2 \text { and } 1-\operatorname{Pr}\left\{\chi_{\nu}^{2}\left(1-\frac{\alpha}{2}\right)<\chi_{\nu}^{2}\right\}=\alpha / 2,
$$

respectively in $\chi^{2}$-distribution with degrees of freedom $\nu$. The relationship (3.22) may be also rewritten as follows:

$$
\begin{gathered}
\operatorname{Pr} .\left\{\hat{\theta}_{L}<E(\hat{\theta})<\hat{\theta}_{L}\right\}=1-\alpha, \\
\hat{\theta}_{L}=n \hat{\theta} / \chi_{\nu}^{2}(\alpha / 2) \quad \text { and } \hat{\theta}_{U}=n \hat{\theta} / \chi_{\nu}^{2}(1-\alpha / 2) .
\end{gathered}
$$

where

Now we are, however, confronted with necessity of using the degrees of freedom $\nu$ of $\chi^{2}$-distribution. For the practical uses, we may and now shall regard the value of the degrees of freedom $\nu$ as $\nu^{\prime \prime}$ defined by $2(n-1)$ $+2 \hat{\delta}^{2} /(n-1+2 \hat{\delta})$, where $\hat{\delta}$ is put as $\left(\hat{\beta}^{2}-\lambda^{-1}\right) S_{\hat{\xi} \hat{\xi}} / 2 \hat{\sigma}^{2}$, in contentment with a precise reason caused by that the values of $\chi_{\nu}^{2}(\alpha / 2) / n$ and $\chi_{\nu}^{2}(1-\alpha / 2) / n$ may scarcely variate, even if there exists a certain small difference, between the $\nu$ and the substitute $\nu^{\prime \prime}$, in case that $\nu>10$, (this is also $n>6$ ), for $\alpha=$ 0.01 or that $\nu>7,(n>4)$, for $\alpha=0.05$ and the assay itself may be designed so as to obtain sufficiently a large value of $\nu$ because of that the values of $S_{\xi \xi}$ and/or the size of $n$ shall be planned relatively large in comparison with the value of $\sigma^{2}$ as described in section 2 .

In conclusion, we may obtain the confidence limits $\hat{\theta}_{C}$ and $\hat{\theta}_{L}$ of $\theta$ in the following way.

$$
\hat{\theta}_{L}=n \hat{\theta} / \chi_{\nu^{\prime \prime}}^{2}\left(\frac{\alpha}{2}\right) \quad \text { and } \quad \hat{\theta}_{C}=n_{\hat{\theta}} / \chi_{\nu^{\prime \prime}}^{2}\left(1-\frac{\alpha}{2}\right),
$$

and thus the confidence limits of $\beta$ are given mediately by

$$
\hat{\beta}_{L}=\hat{\theta}_{L} \pm \sqrt{\hat{\theta}_{L}^{2}+\lambda^{-1}} \quad \text { and } \quad \hat{\beta}_{U}=\hat{\theta}_{V} \pm \sqrt{\hat{\theta}_{L}^{2}+\lambda^{-1}} .
$$

\subsection{Notes on a preliminary estimation of $\lambda$}

Through this paper we are in a situation that the value of $\lambda$ is known to us by a previous information as described in section 2 and that the previous information obtained is independent on the present observations for 
testing and estimation of the relative potency of the test preparation. Now we shall consider the way for obtaining this "previous" information, that is, a value of $\lambda$.

\section{(i) Note on a way to estimate the $\lambda$}

In order to estimate the $\lambda$, let us assume that the repeated trials, $x_{i}$ and $y_{j},\left(i=1,2, \ldots, n_{1} ; j=1,2, \ldots, n_{2}\right)$, are observed at one prescribed doselevel of the standard preparation and let $u_{1}$ and $u_{2}$ be defined by $\sum_{i}\left(x_{i}-\bar{x}\right)^{2}$ $/\left(n_{1}-1\right)$ and $\sum_{j}\left(y_{j}-\bar{y}\right)^{2} /\left(n_{2}-1\right)$ respectively. Then a statistic $\hat{\lambda}$ defined by $\left(n_{2}-3\right) u_{1}^{2} /\left(n_{2}-1\right) u_{2}$ is distributed in $F$-distribution with the pair of degrees of freedom $\left(n_{1}-1, n_{2}-1\right)$ and we obtain that $E(\hat{\lambda})=\lambda$ and $V(\hat{\lambda})=2\left(n_{1}+n_{2}\right.$ $-4) \lambda^{2} /\left(n_{1}-1\right)\left(n_{2}-5\right)$.

Furthermore the confidence limits, $\hat{\lambda}_{V}$ and $\hat{\lambda}_{L}$, of $\lambda$ with a confidence coefficient $1-\alpha$ may be obtained by the following relation,

$$
\operatorname{Pr} .\left\{\frac{n_{2}-1}{n_{2}-3} \bar{\lambda} / F_{n_{1}-1, n_{2}-1}\left(\frac{\alpha}{2}\right)<\lambda<\frac{n_{2}-1}{n_{2}-3} \hat{\lambda} / F_{n_{2}-1, n_{1}-1}\left(\frac{\alpha}{2}\right)\right\}=1-\alpha,
$$

on the basis of that $\left(n_{2}-1\right) \hat{\lambda} /\left(n_{2}-3\right) \lambda$ is distributed in $F$-distribution with the pair of degrees of freedom $\left(n_{1}-1, n_{2}-1\right)$. Namely we may obtain that $\hat{\lambda}_{U}=\frac{n_{2}-1}{n_{2}-3} F_{n_{2}-1, n_{1}-1}\left(\frac{\alpha}{2}\right) \hat{\lambda}$ and $\hat{\lambda}_{L}=\frac{n_{2}-1}{n_{2}-3} F_{n_{1-1, n_{2}-1}}\left(\frac{\alpha}{2}\right) \hat{\lambda}$.

In conclusion, the value of $\lambda$ defined as being known to us in this paper may be applied as $\left(n_{2}-3\right) u_{1} /\left(n_{2}-1\right) u_{2}$ of the preliminary observations or a previous information, because of that the statistic is an unbiassed estimate. And we may indeed apply $\hat{\lambda}_{U}$ and $\hat{\lambda}_{L}$ defined by (3.27), if we would consider such a trial of calculation, to the confidence limits of the relative potency $\beta$ as will substitute $\hat{\beta}_{V}\left(\hat{\theta}_{U}, \hat{\lambda}_{U}^{-1}\right)$ and $\hat{\beta}_{L}\left(\hat{\theta}_{L}, \hat{\lambda}_{L}^{-1}\right)$ for $\hat{\beta}_{U}\left(\hat{\theta}_{U}, \lambda^{-1}\right)$ and $\hat{\beta}_{L}\left(\hat{\theta}_{L}, \lambda^{-1}\right)$ defined by (3.26), but the substitution in this situation may cause the increase the confidence interval of $\beta$ and the confidence coefficient of the interval.

\section{(ii) Note on a relation among the values of $\lambda^{-1}, \theta$ and $\beta$}

Evaluating the expectation and the variance of $\hat{\beta}$, we put both cases, that is, (A) : $\theta^{2}>\lambda^{-1}$ and (B): $\theta^{2}<\lambda^{-1}$ in $\$ 3.2$. This may imply that (A): $|\beta| \leq \lambda^{-1 / 2}(\sqrt{2}-1)$ or $|\beta| \geq \lambda^{-1 / 2}(\sqrt{2}+1)$ and $(\mathrm{B}): \lambda^{-1 / 2}(\sqrt{2}-1)<|\beta|<\lambda^{-1 / 2}(\sqrt{2} \overline{2}$ +1 ) according to the deterministic consideration of comparing a parameter $\theta^{2}$ with a parameter $\lambda^{-1}$, and especially when we may put $\lambda=1$, each case is denoted as (A) : $|\beta|<0.414$ or $|\beta| \geq 2.414$ and (B) : $0.4 .14<|\beta|<2.4 .14$. Supposing that, in our current biological assay, the value of $|\beta|$ may be not so far from one unit by reason of having sufficiently a similar potency of the test preparation as that of the standard preparation. We may meet mostly 
with the case (B), and in such cases as (B) we may be in need of that the better precision of the estimate of $\lambda$ rather desirable than that of $\hat{\theta}$, in a sense of the coefficient of variance.

\section{$\S 4$. Test for the relative potency of test preparation}

The present test problem in our direct assay is principally that whether the potency of a test preparation is equivalent to that of the standard preparation or not. That is to say, our main concern in practical situation is that whether the relative potency $\beta$ of test preparation is equal to one unit or not. We may and now shall consider testing a hypothesis $H_{1}: \beta=1$ against an alternative $H_{2}: \beta \neq 1$, and this test problem is equivalent to testing a hypothesis $H_{1}^{\prime}: \theta=\left(1-\lambda^{-1}\right) / 2$ against an alternative $H_{2}^{\prime}: \theta \neq\left(1-\lambda^{-1}\right) / 2$.

\section{(A) The case when $\lambda=1$ is assumed}

If we may assume especially that $\beta^{2}=\lambda^{-1}$ in the functional relationship on our direct assay, the estimate $\hat{\beta}$ of relative potency is obtained immediately by

$$
\hat{\beta}=\sqrt{\sum_{i}\left(y_{i}-\bar{y}\right)^{2} / \sum_{i}\left(x_{i}-\bar{x}\right)^{2}}
$$

as a maximum likelihood estimate of (3.4). And indeed we are confronted with this case caused by that $\beta^{2}=(\lambda)=1$ to be tested.

The distribution of a statistic $\hat{\beta}$ defined by the square of $\hat{\beta}$ given by (4.1) may be considered to be that of a ratio of both random variables which are distributed in the respective non-central $\chi^{2}$-distribution with the non-centricity parameter $\delta_{1}$ and $\delta_{2}$. Now let us put that

$$
\chi_{y}^{\prime 2}=\sum_{i}\left(y_{i}-\bar{y}\right)^{2} / \sigma^{2} \text { and } \chi_{x}^{\prime 2}=\sum_{i}\left(x_{i}-\bar{x}\right)^{2} / \lambda \sigma^{2},
$$

then $\hat{\beta}^{2}$ is denoted by $\chi_{y}^{\prime 2} / \lambda \chi_{x}^{\prime 2}$ and a probability density of $\hat{\beta}^{2}$ is shown by

$$
f\left(\hat{\beta}^{2}\right) d \hat{\beta}^{2}=\sum_{i=0}^{\infty} \sum_{j=0}^{\infty} \frac{e^{-\delta_{1}} \delta_{1}^{i}}{\Gamma(i+1)} \frac{e^{-\delta_{2}} \delta_{2}^{i}}{\Gamma(j+1)} \frac{\left(\frac{\lambda \hat{\beta}^{2}}{1+\lambda \hat{\beta}^{2}}\right)^{\frac{n-1}{2}+i-1}\left(\frac{1}{1+\lambda \hat{\beta}^{2}}\right)^{\frac{n-1}{2}+j-1}}{\beta\left(\frac{n-1}{2}+i, \frac{n-1}{2}+j\right)} \lambda d \hat{\beta}^{2},
$$

where $\delta_{1}=S_{\xi \xi} / 2 \lambda \sigma^{2}$ and $\delta_{2}=S_{\xi \xi} / 2 \sigma^{2}$. Thus the distribution function of $\hat{\beta}^{2}$ is obtained by

$$
\operatorname{Pr} .\left\{\hat{\beta}^{2}<u\right\}=\sum_{i} \sum_{j}\left(e^{-\delta_{1}} \frac{\delta_{1}^{i}}{i !}\right)\left(e^{-\delta_{2}} \frac{\delta_{2}^{j}}{j !}\right) I_{u}\left(\frac{n-1}{2}+i, \frac{n-1}{2}+j\right),
$$

where $I_{u}\left(\frac{n-1}{2}+i, \frac{n-1}{2}+j\right)$ denotes a beta-distribution with the pair of indices $\left(\frac{n-1}{2}+i, \frac{n-1}{2}+j\right)$ and now $\delta_{1}$ is equal to $\delta_{2}$ on the basis of the assump- 
tion $\lambda=1$.

For the practical uses, testing the hypothesis $H_{1}$ against $H_{2}$ may be performed in the similar mannar as in section 3 by considering an approximation of Patnaik [1].

That is to say, the $\hat{\beta}^{2}$ may be distributed approximately in the $F$-distribution with the pair of degrees of freedom $(\phi, \phi)$, where we put $\phi=(\boldsymbol{n}-1)$ $+4 \delta^{2} /(n-1+4 \delta)$ and $\delta=S_{\xi \xi} / 2 \sigma^{2}$. Then we may and now shall regard the value of the degrees of freedom $\phi$ as $\phi^{\prime}$ defined by $(n-1)+4 \hat{\delta}^{2} /(n-1+4 \hat{\delta})$, where $\hat{\delta}=S_{\hat{\xi} \hat{\xi}} / 2 \hat{\sigma}^{2}$, in contentment with a precise reason that the value of the $\alpha$-percent point of the $F$-distribution with the pair of degrees of freedom $(\phi, \phi)$ may scarcely variate, even if there exists a certain small difference between the $\phi$ and the substitute $\phi^{\prime}$, in case that $\phi>10$ for $\alpha=0.01$ or that $\phi>7$ for $\alpha=0.05$ and the assay itself may be designed so as to obtain sufficiently a large value of $\phi$, because of that the values of $S_{\xi \xi}$ and/or the size of $n$ shall be determined relatively large in comparison with the value of $\sigma^{2}$ as described in section 2. Thus our testing may approximately become a $F$-test with the pair of degrees of freedom $(\phi, \phi)$.

In conclusion, our inference of the relative potency $\beta$ of the case (A) may be performed in the following way.

(i) Let a statistic $\hat{\beta}^{2}$ be given by $\sum_{i}\left(y_{i}-\bar{y}\right)^{2} / \sum_{i}\left(x_{i}-\bar{x}\right)^{2}$.

(ii) We may obtain the estimate $\hat{\beta}$ of the relative potency defined in the following way:

(a) $\hat{\beta}=1$ by accepting $H_{1}$, if $\hat{\beta}^{2}<F_{\phi^{\prime}, \phi^{\prime}}(\alpha)$,

(b) $\hat{\beta}=\hat{\theta} \pm \sqrt{\hat{\theta}^{2}+1}$ by rejecting $H_{1}$, if $\hat{\beta}^{2} \geq F_{\phi^{\prime}, \phi^{\prime}}(\alpha)$,

where $F_{\phi^{\prime}, \phi^{\prime}}(\alpha)$ denotes the $\alpha$-percent point of the $F$-distribution with the pair of degrees of freedom $\left(\phi^{\prime}, \phi^{\prime}\right)$ and $\hat{\theta}$ is given by (3.6).

\section{(B) The case when $\lambda \neq 1$ is assumed}

In the present case we shall make a test problem concerning to $\theta$ instead of $\beta$. For this purpose, having regard to the formula (3.6) and the moments (3.11) of $\hat{\theta}$, we first shall consider the distribution of $\hat{\theta}$ in case when $\beta=1$.

For the practical use, avoiding the excessive complication of the properties (3.11) caused by the original $\hat{\theta}$ in (3.6), we may and now shall consider, like (3.19) in case when $\beta=1$, afresh $\hat{\theta}$ like defined by

$$
\hat{\theta}=\frac{1-\lambda^{-1}}{2}+\frac{m_{02}-\lambda^{-1} m_{20}}{2 S_{\xi \xi}}
$$

with a precise approximation by reason of that $S_{\xi \xi} \gg m_{11}$ and hence $\left(S_{\xi \xi}+\right.$ $\left.m_{11}\right)^{-1} \fallingdotseq S_{\xi \xi}^{-1}$ for $\rho=0$. Indeed, the statistic $\left(m_{02}-\lambda^{-1} m_{20}\right) / 2$ is distributed in $\chi^{2}$-distribution with the degrees of freedom $2(n-1)$ and we may obtain that 
$E\left\{\left(m_{02}-\lambda^{-1} m_{20}\right) / 2\right\}=0$ and $V\left\{\left(m_{02}-\lambda^{-1} m_{20}\right) / 2\right\}=(n-1)\left(\sigma^{2} / n\right)^{2}$ and hence it may be found easily that certain properties obtained from the approximate form (4.5) coheren with those in view of the moments of (3.11) and (3.12).

Therefore we may be found that $\chi_{0}^{\prime 2}$ defined by $n S_{\xi \xi} \hat{\theta} / \sigma^{2}$ is approximately distributed in non-central $\chi^{2}$-distribution with the degrees of freedom $2(n-$ 1) and the non-centricity $\delta=n\left(1-\lambda^{-1}\right) S_{\xi \xi} / 4 \sigma^{2}$.

Thus the present testing hypothesis $H_{1}$ against $H_{2}$ may be also performed by using the approximation of Patnaik [1] with his degrees of freedom on the basis of similar consideration like in the previous (A). Then $\chi_{0}^{2}$ defined by $n S_{\hat{\xi} \xi \hat{\theta}} / C \sigma^{2}$ may be regarded as being distributed in $\chi^{2}$-distribution with the degrees of freedom $\nu^{\prime}$, where we denote that

$$
C=1+\frac{\hat{\delta}}{n-1+\hat{\delta}}, \nu^{\prime}=2(n-1)+2 \hat{\delta}^{2} /(n-1+2 \hat{\delta}) \text { and } \hat{\delta}=n\left(1-\lambda^{-1}\right) S_{\xi \xi} / 4 \sigma^{2} .
$$

Hence we make a statistic $F_{0}$ defined by $\frac{n S_{\hat{\xi} \hat{\xi}} \hat{\theta}}{\nu^{\prime} \sigma^{2}} / \frac{2 n C \hat{\sigma}^{2}}{2(n-1) \sigma^{2}}$, that is, $(n-$ 1) $S_{\hat{\xi} \hat{\xi} \hat{\theta}} / C \nu^{\prime} \hat{\sigma}^{2}$, the $F_{0}$ may be distributed in $F$-distribution with the pair of degrees of freedom $\left(\nu^{\prime}, 2(n-1)\right)$ and we may be able to test a hypothesis $H_{1}$ against the alterative $H_{2}$ by using the statistic $F_{0}$ and by comparing the $F_{0}$ with an $\alpha$-percent point of $F$-distribution with the pair of degrees of freedom $\left(\nu^{\prime}, 2(n-1)\right)$.

While, specially if $n \geq 16$, we know that the distribution of $\sqrt{2 \chi_{0}^{\prime 2} / C}$ is approximated asymptotically to a normal distribution $N\left(\sqrt{ } / \overline{\nu^{\prime}-1}, 1\right)$, where $\nu^{\prime}$ is given by (4.6). Therefore when we make $t_{0}$ defined by $\sqrt{2 S_{\hat{\xi} \xi} \hat{\theta} / C \hat{\sigma}^{2}}$, the $t_{0}$ may be distributed in $t$-distribution with the degrees of freedom $2(n-$ 1) and we may be able to test $H_{1}$ against $H_{2}$ by appling the $t$-test.

In conclusion, our inference of the relative potency $\beta$ of the case (B) may be performed in the following way.

(i) Let a statistic $F_{0}$ be defined by $S_{\hat{\xi} \hat{\xi}} \hat{\theta} / C \hat{\sigma}^{2}$.

(ii) We may obtain the estimate $\hat{\beta}$ of the relative potency $\beta$ defined in the following way:

(a) $\hat{\beta}=1$ by accepting $H_{1}$, if $F_{0}<F_{\nu^{\prime}, 2(n-1)}(\alpha)$,

(b) $\hat{\beta}=\hat{\theta} \pm \sqrt{\hat{\theta}^{2}+\lambda^{-1}}$ by rejecting $H_{1}$, if $F_{0} \geq F_{\nu^{\prime}, 2(n-1)}(\alpha)$,

where $F_{\nu^{\prime}, 2(n-1)}(\alpha)$ denotes an $\alpha$-percent point of the $F$-distribution with the pair of degrees of freedom $\left(\nu^{\prime}, 2(n-1)\right)$.

Specially, in case that $n \geq 16$, the inference of $\beta$ may be obtained as follows :

(i) Let $t_{0}$ be $\sqrt{2 S_{\hat{\xi} \hat{\xi}} \hat{\theta}} / C \hat{\sigma}^{2}$.

(ii) We may obtain $\hat{\beta}$ defined in the following way:

(a) $\hat{\beta}=1$ by accepting $H_{1}$, if $t_{0}<t_{2(n-1)}(\alpha)$,

(b) $\hat{\beta}=\hat{\theta} \pm \sqrt{\hat{\theta}^{2}+\lambda^{-1}}$ by rejecting $H_{1}$, if $t_{0} \geq t_{2(n-1)}(\alpha)$, 
where $t_{2(n-1)}(\alpha)$ denotes an $\alpha$-percent of the $t$-distribution with the degrees of freedom $2(n-1)$.

\section{\$5. Numerical illustrations of the assays of short acting narcotics}

Three short acting narcotics, a derivative $A$ of thiopentalsoda and two derivatives of methylhexabitalsoda $B$ and $C$ have been assayed on the basis of the depth of anesthesia defined by Girndt's method [1], where the preparation $C$ was to be regarded as a standard preparation and $A$ and $B$ were to be compared with $C$ as the test preparation, and the homogeneous puppies selected with care from those of the same ages and the same body weight approximately were used in this assay. The experimental circumstances of this assay were the same conditions as Kido-Asano-Yamamoto [1] in detail and the experimental procedures were performed as follows; a certain dosage of $C$ was first infused as a preliminary operation and secondly a dosage of $A$ or $B$ is infused into a subject until a specified depth-III of Girndt's measurement was observed. In such a situation of our present assay, we have regarded a fluctuation of the critical doses as that caused by preparing and administration of each dose of derivatives. Table 1 shows the administered doses of three narcotics, and table 2 shows the preliminary observations for the value of $\lambda$ between $A$ and $C$.

Table 1. The doses infused into a subject untill the depth-III of anesthesia was observed ( $\mathrm{mg}$. in $\mathrm{kg}$.)

\begin{tabular}{c|cc||cc}
\hline narcotic & $C$ & $B$ & $A$ & $C$ \\
\hline replication & & & & \\
\hline 1 & 12 & $(x)$ & $(y)$ & $(x)$ \\
2 & 4 & 6.5 & 28 & 11 \\
3 & 8.5 & 18 & 13.5 & 26 \\
4 & 8.5 & 9 & 23.5 & 19 \\
5 & 9 & 12 & 21.5 & 16 \\
\hline
\end{tabular}

Table 2. The preliminary observations for the value of $\lambda$ between $A$ and $C$ (at about $10 \mathrm{mg}$.)

\begin{tabular}{c|rrr|rrr}
\hline narcotic & \multicolumn{3}{|c|}{$A$} & \multicolumn{3}{c}{$C$} \\
\hline \multirow{5}{*}{ replications } & 9.44 & 9.34 & 11.38 & 10.49 & 8.90 & 11.05 \\
& 10.79 & 10.15 & 11.72 & 9.21 & 11.79 & 10.13 \\
& 8.86 & 10.47 & 10.44 & 13.43 & 10.33 & 9.48 \\
& 9.91 & 10.97 & 10.60 & 11.29 & 8.89 & 10.96 \\
& 8.69 & 9.76 & 11.87 & 10.41 & 9.52 & 8.35 \\
& 9.80 & 10.29 & 11.27 & 11.67 & 9.34 & 9.57 \\
& 9.44 & 9.15 & 9.72 & 9.23 & 10.44 & 10.29 \\
\hline
\end{tabular}

Now we shall calculate the potencies of $A$ and $B$ relative to $C$ as the numerical examples. 
Example 1: The relative potency of $B$

In this case, we have had good reason to assume that $\lambda=1$, because of that the character of $B$ and $C$ was quite identical in view of the physical considerations. From that $\sum\left(x_{i}-\bar{x}\right)^{2}=32.7, \sum\left(y_{i}-\bar{y}\right)^{2}=94.7, \sum\left(x_{i}-\bar{x}\right)$ $\left(y_{i}-\bar{y}\right)=-46.05$ and the assumption $\lambda=1$, we obtain simply $\hat{\theta}=-0.6732$ and then $\hat{\beta}=-1.879$.

Hence the relative potency $\beta^{*}$ is estimated to be 1.879 , that is, $1 \mathrm{mg}$. of the derivative $B$ is estimated to be equivalent to $1.879 \mathrm{mg}$. of the derivative $C$. As a statement of the findings in an experiment of this kind, the above conclusion may be satisfactory, except that allowance must be made for statistical errors of estimation. We also obtain at the same time that

$$
\begin{array}{llll}
\hat{\mu}=12.4 & \hat{\xi}_{.}=8.4 & \hat{\xi}_{1}=11.6416 & \hat{\xi}_{2}=5.1064 \\
\hat{\xi}_{3}=6.7217 & \hat{\xi}_{4}=9.8323 & \hat{\xi}_{5}=8.6984 . & \hat{\eta}_{1}=6.3090 \\
\hat{\eta}_{2}=18.5887 & \hat{\eta}_{3}=15.5535 & \hat{\eta}_{4}=9.7087 & \hat{\eta}_{5}=11.8393 \\
\hat{\sigma}^{2}=0.8188 . & & &
\end{array}
$$

The confidence limits of the relative potency are calculated after obtaining the numerical results of $\hat{\theta}_{\sigma}$ and $\hat{\theta}_{L}$. Now a value of 14.5 is obtained for the degrees of freedom $\nu^{\prime \prime}$ in (3.25), where $S_{\hat{\xi} \hat{\xi}}=5.2639$ and $\hat{\delta}=8.13$. Then, we may obtain that $\hat{\theta}_{U}=-0.0108$ and $\hat{\theta}_{L}=-0.8270$ with a confidence coefficient 0.99 . Hence, the confidence limits of $\beta$ may be recalculated by (3.26) and obtained as $\hat{\beta} \sigma=-1.0100$ and $\hat{\beta}_{L}=-2.1246$.

Thus the estimated relative potency $\hat{\beta}^{*}, 1.879 \mathrm{mg}$. of the derivative $C$ per mg. of the derivative $B$, is assigned the confidence limits of $1.0100 \mathrm{mg}$. per mg. and $2.1246 \mathrm{mg}$. per mg. .

Furthermore let us consider to test the relative potency of the derivative $B$ as a numerical illustration in the case of (A) in section 4 . The present concern of ours is that whether the potency of the derivative $B$ is equivalent to that of the derivative $C$ or not. From the observations of $B$ and $C$ in table 1 , we may obtain that $\hat{\beta}^{2}=2.896$ and $\phi^{\prime}=6.44 \simeq 6$ with safety. Comparing the $\hat{\beta}^{2}$ with 8.47 of $F_{6,6}(0.99)$, that is, $\hat{\beta}^{2}<F_{6,6}(0.99)$, we cannot find the significance yet and may be unable to reject the hypothesis $H_{0}$ that the potency of $B$ is equivalent to that of $C$ with a confidence coefficient 0.99 .

Example 2: The relative potency of $A$

In this assay, we have had nothing to assume a value of $\lambda$ because of that two derivatives $A$ and $C$ were different each other in view of the chemical and physical considerations. For this, a preliminary investigation given by table 2 was performed with the considerable replications independently of table 1 , and a value of 1.72 was assumed for the $\lambda$. (Indeed a value of 1.72 is an unbiassed estimate of $\lambda$ and we may obtain that $\hat{\lambda}_{U}=4.5263$ and 
$\hat{\lambda}_{L}=0.5310$ as the confidence limits with a probability 0.99 by (3.27)). Thus we obtain that $\hat{\theta}=-0.2057$ and hence $\hat{\beta}=-0.9955$.

Therefore, the relative potency $\hat{\beta}^{*}$ is estimated to be 0.9955 , that is, 1 mg. of the derivative $A$ is estimated to be equivalent to $0.9955 \mathrm{mg}$. of the derivative $C$.

At this time we also obtain the estimates of the parameters as follows:

$\begin{array}{llll}\hat{\mu}=18.8 & \hat{\xi}=20.3 & \hat{\xi}_{1}=28.0854 & \hat{\xi}_{2}=13.2273 \\ \hat{\xi}_{3}=21.3566 & \hat{\xi}_{4}=22.5164 & \hat{\xi}_{5}=16.3143 & \hat{\eta}_{1}=11.0420 \\ \hat{\eta}_{2}=25.8407 & \hat{\eta}_{3}=17.7482 & \hat{\eta}_{4}=16.5937 & \hat{\eta}_{5}=22.7677 \\ \hat{\sigma}^{2}=0.68594373 & & & \end{array}$

The confidence limits $\hat{\theta}_{U}$ and $\hat{\theta}_{L}$ are obtained, as in example 1 , that $\hat{\theta}_{V}$ $=-0.0328$ and $\hat{\theta}_{L}=-0.2524$ with a confidence coefficient 0.99 , where $S_{\hat{\xi} \hat{\xi}}=$ 26.51, $\hat{\delta}=7.915$ and $\nu^{\prime \prime}=14.32$. Hence the confidence limits of $\beta$ are obtained as $\hat{\beta}_{L}=-0.7960$ and $\hat{\beta}_{L}=-1.0556$. Indeed, if we want to such a consideration as $\hat{\lambda}=1.72$, the limits of $\beta$ may be obtained conventionally as -0.5040 and -1.6477 by substituting the values of $\hat{\lambda}_{U}$ and $\hat{\lambda}_{L}$ for 1.72 at $\hat{\beta}_{U}$ and $\hat{\beta}_{L}$ respectively. In this situation we may note worthy that the confidence interval of $\beta$ is enlarged and the confidence coefficient of the interval is increased extremely.

Thus the estimated relative potency $\hat{\beta}^{*}, 0.9955 \mathrm{mg}$. of the derivative $C$ per mg. of the derivative $A$, is assigned the confidence limits of $1.0556 \mathrm{mg}$. and $0.7960 \mathrm{mg}$. per $\mathrm{mg}$.

Testing the relative potency of the derivative $A$, as a numerical illustration in case of (B) in section 4, is performed as follows: We obtain firstly that a value of 0.7694 is obtained for $F_{0}$ defined by $(n-1) S_{\hat{\xi} \hat{\xi}} \hat{\theta} / C \nu^{\prime} \hat{\sigma}^{2}$ where $\nu^{\prime}=22.5, C=1.835$ and $\hat{\delta}=20.24$. Secondly comparing the value of $\boldsymbol{F}_{0}$ with 5.31 of $\boldsymbol{F}_{22,8}(0.99)$, that is $\boldsymbol{F}_{0}<\boldsymbol{F}_{22,8}(0.99)$, we cannot find the significance and may accept the hypothesis $H_{0}$ that the potency of $A$ is equivalent to that of $C$. In conclusion, thus we may be able to decide that the potency of $A$ is the same as $C$ with a confidence coefficient 0.99 .

\section{Summary}

The ordinary method of the biological direct assay has been described by many authors and most investigators being engaged in the biological assay have practiced the method for a long time. It seems, however, to us that the method is in need of large number of subjects and the fiducial limits appling Fieller's theorem are mostly too wide to be useful for the consideration of the relative potency of test preparation, in spite of that the principle of Fieller's theorem itself contains a kind of discussion.

In this paper, an extensive method of the direct assay is proposed and its analysis is shown with the numerical illustrations. Because of that 
our preferable circumstances assumed in section 2 is satisfied naturally as our situation performing the considerable kinds of biological assay, and the analytical method is devised sufficiently from the theoretical and practical viewpoints, it seems that our extensive direct assay may be advantage to estimate and test the potency of test preparation for some direct assays in aid of the above appeals for the improvement of the customary direct assay.

\section{Acknowledgement}

I am very grateful to Prof. T. Kitagawa for his kind suggestions and encouragement concerning the preparation of this manuscript. I am also grateful to Dr. R. Kido for his assistance in the tendering of the data of Table 1 and 2.

KYUSHU UNIVERSITY and SHIONOGI PHARMACEUTICAL CO.

\section{References}

Acton, F. S. : Analysis of Straight-Line Data, John Wiley and Sons (1959).

Berkson, J. : Are there two regressions ?, John. Amer. Stat. Asso., 45 (1950), 168-80.

Bliss, C. I. : The Statistics of Bioassay, Academic Press (1952).

Creasy, M. A. : Confidence limits for the gradient in linear relationship, Jour. Roy. Stat. Soc. Ser. B, 18 (1956), 65-9.

Fieller, E. C. : The biological standardization of insulin, Jour. Roy. Stat. Soc. Suppl., 7 (1940), $1-64$.

Finney, D. J. : Statistical Method in Biological Assay, Charles Griffin and Co. (1952).

- : Experimental Design and its Statistical Basis. Univ. of Chicago Press (1955).

Girndt : Arch. f. Exp. Path. u. Pharmacol., 164 (1932), 118.

Kendall, M. G. : A course in Multivariate Analysis, Charles Griffin and Co. (1957).

Kido, R. Asano, C. and Yamamoto, K. : The bioassay of potency of short acting narcotics, Ann. Rep. Shionogi Research Lab. No. 6 (1956), 203-11. (in Japanese).

Lindley, D. V. : Regression lines and the linear functional relationship, Jour. Roy. Stat. Soc. Suppl, 9 (1947), 218-44.

Patnaik, P. B. : The non-central $\chi^{2-}$ and $F$-distributions and their applications. Biometrika, 36 (1949), 202-232.

Williams, E. J. : Regression analysis, John Wiley and Sons (1959).

Winsor, C. P. : Which regression? Biometric Bull, 2 (1946), 101-9. 